\title{
Compromise programming with Tchebycheff norm for discrete stochastic orders
}

\author{
Sebastian Sitarz
}

Published online: 12 November 2013

(C) The Author(s) 2013. This article is published with open access at Springerlink.com

\begin{abstract}
This paper presents a method of decision making with returns in the form of discrete random variables. The proposed method is based on two approaches: stochastic orders and compromise programming used in multi-objective programming. Stochastic orders are represented by stochastic dominance and inverse stochastic dominance. Compromise programming uses the augmented Tchebycheff norm. This norm, in special cases, takes form of the Kantorovich and Kolmogorov probability metrics. Moreover, in the paper we show applications of the presented methodology in the following problems: projects selections, decision tree and choosing a lottery.
\end{abstract}

Keywords Multi-objective programming · Compromise programming · Tchebycheff norm $\cdot$ Stochastic dominance

\section{Introduction}

The aim of the work is to present a method of decision making in the case of random variables as the returns. The proposed method is reduced to choose a distribution from a finite set of distributions since each decision corresponds to a distribution. The proposed method is based on two approaches: stochastic orders and compromise programming with the augmented Tchebycheff norm. Now, let us look closer at development of these two approaches.

Stochastic dominance, the first approach, is a widely used device in stochastic optimization. General theories of stochastic dominance may be found in the work of Levy (1992) which presents models based on stochastic dominance and expected utility theory. Also, the work of Roman et al. (2006) considers the problem of constructing a portfolio which is nondominated, with respect to second order stochastic dominance; the problem is multi-criteria and is transformed into a single objective problem by using the reference point method. Then, optimization problems with second order inverse stochastic dominance constraints are developed in the paper of Dentcheva and Ruszczyński (2006), however they conclude

S. Sitarz $(\varangle)$

Institute of Mathematics, University of Silesia, ul. Bankowa 14, 40-007 Katowice, Poland

e-mail: ssitarz@math.us.edu.pl 
that the inverse stochastic formulation is mainly of theoretical interest, by providing connections between different utility theories. The works by Sitarz (2010a), Trzaskalik and Sitarz (2007) present the application of stochastic dominance in dynamic programming.

Compromise programming, the second approach, is one of the most frequently applied methods of multi-criteria optimization, both discrete and continuous. This paper uses the approach of Steuer and Choo (1981) who presented an interactive weighted Tchebycheff procedure for multiple objective programming. A problem of weight choice in compromise programming is considered by Ballestero and Romero (1993). Other similar approaches are presented in the work of Carrizosa et al. (1996) which considers so called AS norms in Ideal-Point methods. However, Ballestero (1996) studies the problem of the selection of compromise programming metric in terms of risk aversion. The extension of the compromise programming model into the compromise hypersphere in the case of multi-criteria linear programming is presented by Gass and Roy (2003). Kassem (2008) studies the stability of solutions to multi-objective nonlinear programming problems, using a modified weighted achievement metric based on weighted Tchebycheff metric.

\section{Compromise programming with the augmented Tchebycheff norm}

Our aim is to choose a distribution from a finite set of distributions since each decision corresponds to a distribution. In order to realize it, we use the augmented Tchebycheff norm. The general form of the augmented Tchebycheff norm allows us to use the Kantorovich and Kolmogorov probability metrics for distribution functions.

One of the approaches to compromise programming is presented in the work of Steuer and Choo (1981), where the augmented weighted Tchebycheff norm, for measuring the distance between any criteria vector $\mathbf{z}$ and the ideal vector $\mathbf{z}^{*}$, is defined as follows:

$$
\left\|\left|\mathbf{z}^{*}-\mathbf{z}\right|\right\|_{\infty}^{\lambda}=\max _{k}\left\{\lambda_{k}\left|z_{k}^{*}-z_{k}\right|\right\}+\rho \sum_{k}\left|z_{k}^{*}-z_{k}\right|
$$

where $\rho$ is fixed scalar $(\rho \geq 0)$ and $\lambda$ is weight vector $(\lambda \geq 0)$.

The idea of compromise programming is to find a vector $\mathbf{z}$ in the criteria space which minimizes the distance to the ideal vector:

$$
\min _{\mathbf{z} \in Z}\left\|\left|\mathbf{z}^{*}-\mathbf{z}\right|\right\|_{\infty}^{\lambda}
$$

where $Z$ is a feasible region in criteria space.

\subsection{Notation for distribution function}

The application of the augmented weighted Tchebycheff norm in the case of random variables as the returns is presented in Sects. 3 and 4. In these sections we consider the finite family of random variables $\left\{\xi_{i}: i \in I\right\}$. Moreover, we assume that the following set

$$
X=\left\{x: \exists_{i \in I} P\left(\xi_{i}=x\right)>0\right\}
$$

is finite. It means that we are able to enumerate the elements of the set $X$

$$
X=\left\{x_{1}, x_{2}, \ldots, x_{n}\right\}
$$

where $x_{s}<x_{t}$ for $s<t$.

We use the following notation for the given random variable: 
$F_{\xi}(x)=\mathbb{P}(\xi \leq x)$ - the right-continuous distribution function of the random variable $\xi$

$F_{\xi}^{(1)}(x)=F_{\xi}(x)$,

$F_{\xi}^{(k)}(x)=\int_{-\infty}^{x} F^{(k-1)}(t) d t$, for $k \geq 2$

In the following subsections, we are going to present the connections between the augmented Tchebycheff norm for discrete random variables and probability metrics.

\subsection{Kolmogorov metric}

We formulate the condition for parameters of the augmented Tchebycheff norm, for which it corresponds to Kolmogorov metric often used in stochastic analysis, for example in the work by Gibbs and Su (2002). We use the following notation:

$$
\mathbf{z}^{i}=\left[F_{\xi_{i}}\left(x_{1}\right), F_{\xi_{i}}\left(x_{2}\right), \ldots, F_{\xi_{i}}\left(x_{n}\right)\right] .
$$

By putting $\rho=0$ and for each index $i: \lambda_{i}=1$ we obtain the following form of the Tchebycheff norm

$$
\left\|\left|\mathbf{z}^{i}-\mathbf{z}^{j}\right|\right\|_{\infty}^{\lambda}=\max _{k}\left|z_{k}^{i}-z_{k}^{j}\right|
$$

Moreover, by using discrete form of the considered random variables, we obtain:

$$
\max _{k}\left|z_{k}^{i}-z_{k}^{j}\right|=\sup _{x}\left|F_{\xi_{i}}(x)-F_{\xi_{j}}(x)\right|
$$

The above formula is equivalent to distance measurement between two distribution functions by using Kolmogorov metric. Thus the augmented Tchebycheff norm with $\rho=0$ and $\lambda_{i}=1$ describes the Kolmogorov metric.

\subsection{Kantorovich metric}

We formulate the condition for parameters of the Tchebycheff norm, for which it corresponds to Kantorovich metric, for example presented in the work by Gibbs and Su (2002).

We assume that the elements of the set $X$ satisfy the following condition:

$$
x_{2}-x_{1}=x_{3}-x_{2}=\cdots=x_{n}-x_{n-1}=\text { const }
$$

By putting $\rho=1 /$ const and for each index $i: \lambda_{i}=0$ we obtain the following form of the augmented Tchebycheff norm:

$$
\left\|\left|\mathbf{z}^{i}-\mathbf{z}^{j}\right|\right\|_{\infty}^{\lambda}=(1 / \text { const }) \sum_{k}\left|z_{k}^{i}-z_{k}^{j}\right|=\int_{-\infty}^{\infty}\left|F_{\xi_{i}}(x)-F_{\xi_{j}}(x)\right| d x
$$

The above formula is equivalent to distance measurement between two distribution functions by using Kantorovich metric. Thus, we have shown the connections between the augmented Tchebycheff norm and Kantorovich metric.

\subsection{Sum of Kolmogorov and Kantorovich metrics}

It is possible to use the sum of Kolmogorov and Kantorovich metrics. Under the following assumptions presented earlier in Sects. 2.2 and 2.3: 


$$
\begin{aligned}
& x_{2}-x_{1}=x_{3}-x_{2}=\cdots=x_{n}-x_{n-1}=\text { const } \\
& \rho=1 / \text { const } \\
& \quad \text { for each index } i: \lambda_{i}=1
\end{aligned}
$$

we obtain the following form of the augmented Tchebycheff norm:

$$
\left\|\left|\mathbf{z}^{i}-\mathbf{z}^{j}\right|\right\|_{\infty}^{\lambda}=\sup _{x}\left|F_{\xi_{i}}(x)-F_{\xi_{j}}(x)\right|+\int_{-\infty}^{\infty}\left|F_{\xi_{i}}(x)-F_{\xi_{j}}(x)\right| d x
$$

The above equation presents the sum of Kolmogorov and Kantorovich metrics.

Remark 2.1 In the work by Stoyanov et al. (2012) some ways of metrization of higher orders of stochastic dominance by means of the Levy quasi-semidistance are presented. However, the metrics presented by Stoyanov et al. (2012) are not suitable for the augmented Tchebycheff norm in the case of higher orders of stochastic dominance presented in this paper.

\section{Compromise programming for stochastic dominance model}

We are going to use the concept of compromise programming with the augmented Tchebycheff norm to stochastic dominance model. First, we present stochastic dominance model (Sect. 3.1) and next we use the augmented Tchebycheff norm (Sect. 3.2).

\subsection{Stochastic dominance model}

In this subsection, we use the works by Rolski (1976), Ogryczak and Ruszczyński (1997).

Relations of $k$-th order stochastic dominance are defined as follows:

$$
\begin{aligned}
& \xi_{1} \leq^{(k)} \xi_{2} \quad \Leftrightarrow \quad \forall_{x \in \mathbb{R}} F_{\xi_{1}}^{(k)}(x) \geq F_{\xi_{2}}^{(k)}(x) \\
& \xi_{1}<{ }^{(k)} \xi_{2} \quad \Leftrightarrow \quad \xi_{1} \leq^{(k)} \xi_{2} \wedge F_{\xi_{1}}^{(k)} \neq F_{\xi_{2}}^{(k)}
\end{aligned}
$$

We assume that we consider random variables for which the integrals mentioned above exist. In special cases when $k=1(k=2)$, the first (second) order stochastic dominance is usually denoted as FSD or $\leq_{\text {FSD }}(\mathrm{SSD}$ or $\leq \mathrm{SSD})$.

\subsection{The augmented Tchebycheff norm for stochastic dominance}

We build a vector $\mathbf{z}^{i}$ connected with discrete random variables $\xi_{i}$, in the following way:

$$
{ }^{(1)} \mathbf{z}^{i}=\mathbf{z}^{i}=\left[z_{1}^{i}, z_{2}^{i}, \ldots, z_{n}^{i}\right]=\left[F_{\xi_{i}}\left(x_{1}\right), F_{\xi_{i}}\left(x_{2}\right), \ldots, F_{\xi_{i}}\left(x_{n}\right)\right],
$$

Continuing the procedure, we build the vectors ${ }^{(k)} \mathbf{z}^{i}$ :

$$
{ }^{(k)} \mathbf{z}^{i}=\left[{ }^{(k)} z_{1}^{i},{ }^{(k)} z_{2}^{i}, \ldots,{ }^{(k)} z_{n}^{i}\right]=\left[F_{\xi_{i}}^{(k)}\left(x_{1}\right), F_{\xi_{i}}^{(k)}\left(x_{2}\right), \ldots, F_{\xi_{i}}^{(k)}\left(x_{n}\right)\right]
$$

We denote the finite feasible region of the program (1) by

$$
{ }^{(k)} Z=\left\{{ }^{(k)} \mathbf{z}^{i}: i \in I\right\}
$$


We define an ideal criterion $\mathbf{z}^{*}$ vector for FSD as follows:

$$
{ }^{(1)} z_{m}^{*}=z_{m}^{*}=\min _{i \in I} z_{m}^{i}
$$

The above construction for the ideal vector is based on the form of the relation for FSD: the lower value of the distribution, the better. Thus, the ideal vector is presented by the minimum of the considered values for distribution. Such construction is well known from the classical multi-criteria decision making techniques (see for example Steuer and Choo 1981).

\section{Theorem 1}

1. Vector $\mathbf{z}^{*}$ generates the following random variable $\xi^{*}$ :

$$
P\left(\xi^{*}=x\right)= \begin{cases}z_{1}, & x=x_{1} \\ z_{m}-z_{m-1}, & x=x_{m} \wedge m \in\{2, \ldots, n\} \\ 0, & \text { otherwise }\end{cases}
$$

2. Using $\xi^{*}$, for each random variable $\xi_{i}$, we obtain:

$$
\forall_{k} \quad \xi_{i} \leq^{(k)} \xi^{*}
$$

3. For every two random variables $\xi_{i}$, $\xi_{j}$ it holds:

$$
\xi_{i} \leq^{(k)} \xi_{j} \Rightarrow\left\|\left.\right|^{(k)} \mathbf{z}^{*}-{ }^{(k)} \mathbf{z}^{i}\left|\left\|_{\infty}^{\lambda} \geq\right\|\right|^{(k)} \mathbf{z}^{*}-{ }^{(k)} \mathbf{z}^{j} \mid\right\|_{\infty}^{\lambda}
$$

where $^{(k)} \mathbf{z}^{i}$ and ${ }^{(k)} \mathbf{z}^{j}$ are connected with random variables $\xi_{\mathrm{i}}, \xi_{\mathrm{j}}$.

4. Let ${ }^{(k)} Z^{*}$ denote a set of minimal solutions of program $(1)$, and ${ }^{(k)} Z^{N}$ denote a set of nondominated criterion vectors in ${ }^{(k)} Z$. Then

$$
{ }^{(k)} Z^{*} \cap{ }^{(k)} Z^{N} \neq \varnothing
$$

It means that a minimal solution of program (1) exists, which is nondominated in ${ }^{(k)} Z$.

5. If $\rho>0$ and $\lambda>0$, then

$$
{ }^{(k)} Z^{*} \subset{ }^{(k)} Z^{\mathrm{N}}
$$

It means that every minimal solution of program (1) is nondominated in ${ }^{(k)} Z$.

Proof See the Appendix.

\section{Compromise programming for inverse stochastic dominance model}

In this section, we are going to use the concept of compromise programming with the augmented Tchebycheff norm to inverse stochastic dominance model. First, we present inverse stochastic dominance model (Sect. 4.1) and next we use the augmented Tchebycheff norm (Sect. 4.2). 


\subsection{Inverse stochastic dominance model}

In this subsection we use the works by Rolski (1976), Ogryczak and Ruszczyński (1997). We use the following notation:

$$
\begin{aligned}
& \bar{F}_{\xi}(x)=1-F_{\xi}(x), \bar{F}_{\xi}(x) \\
& \bar{F}_{\xi}^{(1)}(x)=\bar{F}_{\xi}(x), \\
& \bar{F}_{\xi}^{(k)}(x)=\int_{x}^{\infty} \bar{F}_{\xi}^{(k-1)}(t) d t, \quad \text { for } k \geq 2
\end{aligned}
$$

Relations of $k$-th order inverse stochastic dominance are defined as follows:

$$
\begin{aligned}
& \xi_{1} \leq^{(\bar{k})} \xi_{2} \quad \Leftrightarrow \quad \forall_{x \in \mathbb{R}} \bar{F}_{\xi_{1}}^{(k)}(x) \leq \bar{F}_{\xi_{2}}^{(k)}(x) \\
& \xi_{1}<{ }^{(\bar{k})} \xi_{2} \quad \Leftrightarrow \quad \xi_{1} \leq^{(k)} \xi_{2} \wedge \bar{F}_{\xi_{1}}^{(k)} \neq \bar{F}_{\xi_{2}}^{(k)}
\end{aligned}
$$

The relation of first order inverse stochastic dominance is equivalent to the relation of first order stochastic dominance. Hence, a relation of first inverse stochastic dominance is not considered. In special cases when $k=2(k=3)$, the second (third) order inverse stochastic dominance is usually denoted as SISD or $\leq_{\text {SISD }}$ (TISD or $\leq_{\text {TISD }}$ ).

Remark 4.1 We present stochastic orders in a general way. However, the theoretical and practical importance of these orders is a departure from one another. The first order stochastic dominance guarantees that the expected utility of better random variable is greater than that of worst random variable for all increasing utility functions. For the second order stochastic dominance we have similar property for all increasing concave utility functions. In contrast, second order stochastic dominance not only assumes increasing utility but it also assumes that utility must increase at a decreasing rate (Copeland and Weston 1988).

\subsection{The augmented Tchebycheff norm for inverse stochastic dominance}

We build a vector $\overline{\mathbf{z}}^{i}$ connected with discrete random variables $\xi_{i}$ in the following way:

$$
{ }^{(1)} \overline{\mathbf{z}}^{i}=\overline{\mathbf{z}}^{i}=\left[\bar{z}_{1}^{i}, \bar{z}_{2}^{i}, \ldots, \bar{z}_{n}^{i}\right]=\left[\bar{F}_{\xi_{i}}\left(x_{1}\right), \bar{F}_{\xi_{i}}\left(x_{2}\right), \ldots, \bar{F}_{\xi_{i}}\left(x_{n}\right)\right],
$$

Continuing the procedure, we build vectors ${ }^{(k)} \overline{\mathbf{z}}^{i}$ :

$$
{ }^{(k)} \overline{\mathbf{z}}^{i}=\left[{ }^{(k)} \bar{z}_{1}^{i},{ }^{(k)} \bar{z}_{2}^{i}, \ldots,{ }^{(k)} \bar{z}_{n}^{i}\right]=\left[\bar{F}_{\xi_{i}}^{(k)}\left(x_{1}\right), \bar{F}_{\xi_{i}}^{(k)}\left(x_{2}\right), \ldots, \bar{F}_{\xi_{i}}^{(k)}\left(x_{n}\right)\right]
$$

We denote the finite feasible region of the Tchebycheff program (1) by

$$
{ }^{(k)} \bar{Z}=\left\{{ }^{(k)} \overline{\mathbf{z}}^{i}: i \in I\right\} .
$$

We define an ideal vector $\overline{\mathbf{z}}^{*}$ in this case as follows:

$$
{ }^{(1)} \bar{z}_{m}^{*}=\bar{z}_{m}^{*}=\max _{i \in I} \bar{z}_{m}^{i}
$$

The above construction for the ideal vector is based on the form of the relation for inverse stochastic model: the greater value of the distribution, the better. Thus, the ideal vector is 
presented by the maximum of the considered values for distribution. Such construction is well known from the classical multi-criteria decision making techniques (see for example Steuer and Choo 1981).

\section{Theorem 2}

1. Vector $\overline{\mathbf{z}}^{*}$ generates the following random variable $\bar{\xi}^{*}$ :

$$
P\left(\bar{\xi}^{*}=x\right)= \begin{cases}1-\bar{z}_{1}, & x=x_{1} \\ \bar{z}_{m-1}-\bar{z}_{m}, & x=x_{m} \wedge m \in\{2, \ldots, n\} \\ 0, & \text { otherwise }\end{cases}
$$

2. Using $\bar{\xi}^{*}$, for each random variable $\xi_{i}$, we obtain:

$$
\forall_{k} \quad \xi_{i} \leq^{(\bar{k})} \bar{\xi}^{*}
$$

3. For every two random variables $\xi_{i} \xi_{j}$ it holds:

$$
\xi_{i} \leq^{(\bar{k})} \xi_{j} \Rightarrow\left\|\left.\right|^{(k)} \overline{\mathbf{z}}^{*}-{ }^{(k)} \overline{\mathbf{z}}^{i}\left|\left\|_{\infty}^{\lambda} \geq\right\|\right|^{(k)} \overline{\mathbf{z}}^{*}-{ }^{(k)} \overline{\mathbf{z}}^{j} \mid\right\|_{\infty}^{\lambda},
$$

where ${ }^{(k)} \overline{\mathbf{z}}^{i},{ }^{(k)} \overline{\mathbf{z}}^{j}$ are connected with random variables $\xi_{i}, \xi_{j}$

4. Let ${ }^{(k)} \bar{Z}^{*}$ denote a set of minimal solutions of program (1), and ${ }^{(k)} \bar{Z}^{N}$ denote a set of nondominated criterion vectors in ${ }^{(k)} \bar{Z}$. Then

$$
{ }^{(k)} \bar{Z}^{*} \cap{ }^{(k)} \bar{Z}^{N} \neq \varnothing
$$

It means that a minimal solution of program (1) exists, which is nondominated in ${ }^{(k)} \bar{Z}$.

5. If $\rho>0$ and $\lambda>0$, then

$$
{ }^{(k)} \bar{Z}^{*} \subset{ }^{(k)} \bar{Z}^{N}
$$

It means that every minimal solution of program (1) is nondominated in ${ }^{(k)} \bar{Z}$.

Proof Analogously, as in the case of Theorem 1.

\section{Procedure}

The aim of the proposed procedure is to choose a distribution from a finite set of distributions since each decision corresponds to a distribution. The method is based on stochastic orders and the augmented Tchebycheff norm. The procedure is as follows:

Step 1. Establish feasible decisions and corresponding random variables.

Step 2. Establish stochastic order (one of the presented in Sects. 3 and 4).

Step 3. Build an ideal point (respectively: ${ }^{(k)} \mathbf{z}^{*},{ }^{(k)} \overline{\mathbf{z}}^{*}$, see Sects. 3 and 4).

Step 4. Establish the norm, i.e. set scalar $\rho \geq 0$ and weight vector $\lambda \geq 0$ (see Sect. 2).

Step 5. Solve program (1) and obtain the preferable decision as the vector which is closest to an ideal vector, $\left({ }^{(k)} Z^{*},{ }^{(k)} \bar{Z}^{*}\right.$ respectively).

The above procedure is characterized by the following interesting properties: 
Table 1 Data for the example in Sect. 6.1

\begin{tabular}{lllllllllllllllllllll}
\hline$i$ & 1 & 2 & 3 & 4 & 5 & 6 & 7 & 8 & 9 & 10 & 11 & 12 & 13 & 14 & 15 & 16 & 17 & 18 & 19 & 20 \\
\hline$P\left(\xi_{i}=1\right)$ & & & .4 & .2 & & .6 & & & .2 & .4 & .2 & & & .2 & .4 & & .6 & & \\
$P\left(\xi_{i}=2\right)$ & & & .4 & & & & & & .2 & .2 & .4 & .2 & & .2 & .6 & & .4 & .2 & & \\
$P\left(\xi_{i}=3\right)$ & .2 & .4 & .2 & .4 & & .2 & .4 & & .4 & .4 & .2 & & .2 & & & .2 & & .2 & .2 & .2 \\
$P\left(\xi_{i}=4\right)$ & .6 & 0 & & .4 & .6 & .2 & .2 & .8 & .2 & & .2 & .6 & .4 & .6 & & .2 & & .6 & .4 & .6 \\
$P\left(\xi_{i}=5\right)$ & .2 & .6 & & & .4 & & .2 & .2 & & & & .2 & .4 & & & .6 & & & .4 & .2 \\
\hline
\end{tabular}

\section{Properties of procedure}

P1. There exists an element in ${ }^{(k)} Z^{*}\left[{ }^{(k)} \bar{Z}^{*}\right]$ which is nondominated in ${ }^{(k)} Z\left[{ }^{(k)} \bar{Z}\right]$.

P2. Moreover for the stochastic dominance model, if $\rho>0$ and $\lambda>0$, then each element in ${ }^{(k)} Z^{*}\left[{ }^{(k)} \bar{Z}^{*}\right]$ is nondominated in ${ }^{(k)} Z\left[{ }^{(k)} \bar{Z}\right]$.

P3. Each nondominated element in ${ }^{(k)} Z\left[{ }^{(k)} \bar{Z}\right]$ may be obtained as a minimal solution of program (1) (i.e. element in ${ }^{(k)} Z^{*}\left[{ }^{(k)} \bar{Z}^{*}\right]$ ) when $\rho$ and $\lambda>0$ are properly chosen.

\section{Proof}

Property P1 results from 4. of Theorems 1 and 2.

Property P2 results from 5. of Theorems 1 and 2.

Property P3 results from Steuer and Choo (1981, Theorem 3.7).

\section{Applications}

We propose three applications of the presented theory: the project selection problem, the decision tree problem and choosing a lottery problem. We use in these examples the Kantorovich metric, Kolmogorov metric, and the sum of these metrics, respectively.

\subsection{Project selection problem}

To illustrate our procedure (Sect. 5), let us consider the project selection problem presented by Nowak (2007). Twenty proposals are evaluated with respect to the second attribute which is the second one of three attributes considered by Nowak (2007) in his initial example. The probabilities used in this example are given directly from the work by Nowak (2007).

Step 1. We consider twenty random variables for each project. The probabilities characterizing these variables are presented in Table 1 (they are given by Nowak 2007).

Step 2. We use first order stochastic dominance (FSD) as stochastic order.

Step 3. Vectors $\mathbf{z}^{i}$ built for considered random variables are presented in Table 2, where the ideal vector $\mathbf{z}^{*}$ is also given (the ideal vector is given by the formula from Sect. 3 as a minimum of $\mathbf{z}^{i}$. In this case the vector means an ideal distribution function-the best distribution function built from the considered distribution functions.).

Step 4. We use the augmented Tchebycheff norm with the following parameters:

$$
\begin{aligned}
& \rho=1, \\
& \lambda=0=[0,0,0,0,0] .
\end{aligned}
$$


Table 2 Vectors $\mathbf{z}^{i}, \mathbf{z}^{*}$ and the values of the Kolmogorov metric in the example in Sect. 6.1

\begin{tabular}{|c|c|c|c|c|c|c|c|c|c|c|c|c|c|c|c|c|c|c|c|c|c|}
\hline$i$ & 1 & 2 & 3 & 4 & 5 & 6 & 7 & 8 & 9 & 10 & 11 & 12 & 13 & 14 & 15 & 16 & 17 & 18 & 19 & 20 & $\mathbf{z}^{*}$ \\
\hline$z_{1}^{i}$ & 0 & 0 & .4 & .2 & 0 & .6 & 0 & 0 & .2 & .4 & .2 & 0 & 0 & .2 & .4 & 0 & .6 & 0 & 0 & 0 & 0 \\
\hline$z_{2}^{i}$ & 0 & 0 & .8 & .2 & 0 & .6 & 0 & 0 & .4 & 6 & .6 & .2 & 0 & .4 & 1 & 0 & 1 & .2 & 0 & 0 & 0 \\
\hline$z_{3}^{i}$ & .2 & .4 & 1 & .6 & 0 & .8 & .4 & 0 & .8 & 1 & .8 & .2 & .2 & .4 & 1 & .2 & 1 & .4 & .2 & .2 & 0 \\
\hline$z_{4}^{i}$ & .8 & .4 & 1 & 1 & .6 & 1 & .8 & .8 & 1 & 1 & 1 & .8 & .6 & 1 & 1 & .4 & 1 & 1 & .6 & .8 & .4 \\
\hline$z_{5}^{i}$ & 1 & 1 & 1 & 1 & 1 & 1 & 1 & 1 & 1 & 1 & 1 & 1 & 1 & 1 & 1 & 1 & 1 & 1 & 1 & 1 & 1 \\
\hline \multicolumn{22}{|c|}{ Values of the Kolmogorov metric } \\
\hline & 6 & .4 & 2.8 & 1.6 & .2 & 2.6 & .8 & .4 & 2 & 2.6 & 2.2 & .8 & .4 & 1.6 & 3 & .2 & 3.2 & 1.2 & .4 & 6 & \\
\hline
\end{tabular}

Fig. 1 Special Instrument Products decision tree (Kirkwood 2002)

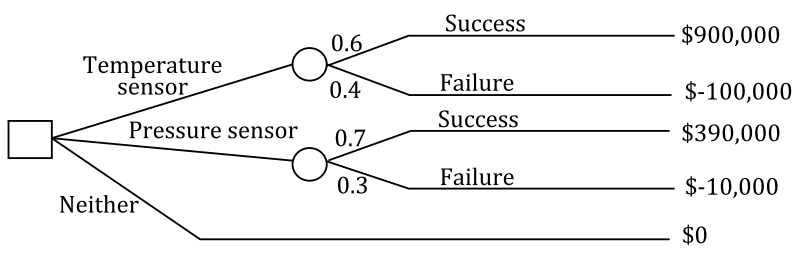

Let us notice that all parameters satisfy the assumptions of the Kantorovich metric presented in Sect. 2.3. Thus, the augmented Tchebycheff norm means using Kantorovich metric in this example.

Step 5. Table 2 presents the values of the augmented Tchebycheff norm for succeeding vectors $\mathbf{z}^{i}$. Vectors $\mathbf{z}^{5}$ and $\mathbf{z}^{16}$ are the closest to the ideal vector, therefore the preferable projects are the projects 5 and 16 connected with $\xi_{5}$ and $\xi_{16}$.

\subsection{Decision tree problem}

The presented procedure in Sect. 5 can be applied in the decision tree problems. To illustrate this application we consider an example of the product decision problem from work by Kirkwood (2002). To absorb some short-term excess production capacity at its Arizona plant, Special Instrument Products is considering a short manufacturing run for either of two new products, a temperature sensor or a pressure sensor. The market for each product is known if the products can be successfully developed. However, there is some chance that it will not be possible to successfully develop them. Revenue of $\$ 1,000,000$ would be realized from selling the temperature sensor and revenue of $\$ 400,000$ would be realized from selling the pressure sensor. Both of these amounts are net of production cost but do not include development cost. If development is unsuccessful for a product, then there will be no sales, and the development cost will be totally lost. Development cost would be $\$ 100,000$ for the temperature sensor and $\$ 10,000$ for the pressure sensor. The probability of development success is 0.6 for the temperature sensor and 0.7 for the pressure sensor. Figure 1 presents a diagram for this problem.

We proceed the procedure from Sect. 5.

Step 1. We consider the set of three discrete random variables connected with decisions:

- choosing a temperature sensor $\left(\xi_{1}\right)$

- choosing a pressure sensor $\left(\xi_{2}\right)$

- neither $\left(\xi_{3}\right)$ 
Table 3 Description of random variables in the example in Sect. 6.2

Table 4 Vectors $\mathbf{z}^{i}, \mathbf{z}^{*}$ and values of the Kolmogorov metric in the example in Sect. 6.2

\begin{tabular}{llll}
\hline & $\xi_{1}$ & $\xi_{2}$ & $\xi_{3}$ \\
\hline$P\left(\xi_{i}=-100000\right)$ & 0.4 & 0 & 0 \\
$P\left(\xi_{i}=-10000\right)$ & 0 & 0.3 & 0 \\
$P\left(\xi_{i}=0\right)$ & 0 & 0 & 1 \\
$P\left(\xi_{i}=390000\right)$ & 0 & 0.7 & 0 \\
$P\left(\xi_{i}=900000\right)$ & 0.6 & 0 & 0 \\
\hline
\end{tabular}

\begin{tabular}{llll}
\hline $\mathbf{z}^{*}$ & $\mathbf{z}^{1}$ & $\mathbf{z}^{2}$ & $\mathbf{z}^{3}$ \\
\hline 0 & 0.4 & 0 & 0 \\
0 & 0.4 & 0.3 & 0 \\
0.3 & 0.4 & 0.3 & 1 \\
0.4 & 0.4 & 1 & 1 \\
1 & 1 & 1 & 1 \\
The Kolmogorov metric & 0.4 & 0.6 & 0.7 \\
\hline
\end{tabular}

The probabilities characterizing these variables are presented in Table 3.

Step 2. We use the first order stochastic dominance (FSD) as stochastic order.

Step 3. Vectors $\mathbf{z}^{i}$ built for considered random variables are presented in Table 4, where the ideal vector $\mathbf{z}^{*}$ is also given. The ideal vector is given by the formula from Sect. 3 as a minimum of $\mathbf{z}^{i}$ - the best distribution function built from the considered distribution functions.

Step 4. We use the augmented Tchebycheff norm with the following parameters:

$$
\begin{aligned}
& \rho=0, \\
& \lambda=1=[1,1,1,1,1] .
\end{aligned}
$$

It means that we use the Kolmogorov metric for distributions.

Step 5. Table 4 presents the values of the augmented Tchebycheff norm for succeeding vectors $\mathbf{z}^{i}$. Vector $\mathbf{z}^{1}$ is closest to the ideal vector, therefore the preferable random variable is $\xi_{1}$ and the best decision: choosing a temperature sensor.

\subsection{Choosing a lottery}

The presented earlier approach can be also applied to the problem of choosing a lottery. To illustrate this case, we consider an example of choosing a lottery from the set of five lotteries. The possible wins and probabilities are given in Table 5.

We proceed the procedure from Sect. 5.

Step 1. We consider five random variables for each lottery. The probabilities characterizing these variables are presented in Table 5.

Step 2. We use the second order inverse stochastic dominance (SISD).

Step 3. Vectors ${ }^{(2)} \overline{\mathbf{z}}^{i}$ for succeeding random variables are presented in Table 6 , where the ideal vector ${ }^{(2)} \overline{\mathbf{z}}^{*}$ is also given as a maximum of ${ }^{(2)} \overline{\mathbf{z}}^{i}$. The ideal vector ${ }^{(2)} \overline{\mathbf{z}}^{*}$ means the best distribution function built from the considered distribution functions in the sense of SISD model. 
Table 5 Description of lotteries in the example in Sect. 6.3

\begin{tabular}{llllll}
\hline & $L_{1}$ & $L_{2}$ & $L_{3}$ & $L_{4}$ & $L_{5}$ \\
\hline$\$ 1$ & 0.05 & 0 & 0.1 & 0 & 0 \\
$\$ 2$ & 0.15 & 0.2 & 0 & 0.05 & 0.5 \\
$\$ 3$ & 0.1 & 0 & 0 & 0.3 & 0 \\
$\$ 4$ & 0.2 & 0.4 & 0.7 & 0.4 & 0.5 \\
$\$ 5$ & 0.5 & 0.4 & 0.2 & 0.25 & 0 \\
\hline
\end{tabular}

Table 6 Considered vectors and values of metric in the example in Sect. 6.3

\begin{tabular}{llllll}
\hline${ }^{(2)} \overline{\mathbf{z}}^{1}$ & ${ }^{(2)} \overline{\mathbf{z}}^{2}$ & ${ }^{(2)} \overline{\mathbf{z}}^{3}$ & ${ }^{(2)} \overline{\mathbf{z}}^{4}$ & ${ }^{(2)} \overline{\mathbf{z}}^{5}$ & ${ }^{(2)} \overline{\mathbf{z}}^{*}$ \\
\hline 0.05 & 0 & 0.1 & 0 & 0 & 0.1 \\
0.2 & 0.2 & 0.1 & 0.05 & 0.5 & 0.5 \\
0.3 & 0.2 & 0.1 & 0.35 & 0.5 & 0.5 \\
0.5 & 0.6 & 0.8 & 0.75 & 1 & 1 \\
1 & 1 & 1 & 1 & 1 & 1 \\
1.55 & 1.5 & 1.4 & 1.4 & 0.2 & \|\|$\left.^{(2)} \overline{\mathbf{z}}^{*}-{ }^{(2)} \overline{\mathbf{z}}^{i} \mid \|_{\infty}^{\lambda,}, 1, \lambda\right]$ \\
\hline
\end{tabular}

Step 4. We use the augmented Tchebycheff norm with the following parameters:

$$
\begin{aligned}
& \rho=1, \\
& \lambda=1=[1,1,1,1,1] .
\end{aligned}
$$

Thus, in this case, the augmented Tchebycheff norm means using the sum of Kantorovich and Kolmogorov metrics.

Step 5. Table 6 presents the values of the function $\left\|\left.\right|^{(2)} \overline{\mathbf{z}}^{*}-{ }^{(2)} \overline{\mathbf{z}}^{i} \mid\right\|_{\infty}^{\lambda}$ for succeeding vectors ${ }^{(2)} \overline{\mathbf{z}}^{i}$. Vector ${ }^{(2)} \overline{\mathbf{z}}^{5}$ is the closest to the ideal vector, therefore the preferable lottery is $L_{5}$.

\section{Conclusions and further research}

The work has proposed a new method of decision making based on the augmented Tchebycheff norm for distribution selection from a set of distributions. The general form of the augmented Tchebycheff norm allows us to use $1^{1}$-norm in the case of Kantorovich and Kolmogorov probability metrics.

Moreover, we have used stochastic dominance and inverse stochastic. In future, it is worth considering the following problems:

- a case of continuous random variables

- an interactive version of the proposed method for stochastic dominance model.

- a comparison optimization properties of the augmented Tchebycheff norm, classic Zelezny-Yu compromise programming metrics and others.

- using sensitivity analysis of the model by multi-objective methods (Sitarz 2008, 2010b, 2012). 
Open Access This article is distributed under the terms of the Creative Commons Attribution License which permits any use, distribution, and reproduction in any medium, provided the original author(s) and the source are credited.

\section{Appendix: Proof of Theorem 1}

1. We will check that $\xi^{*}$ is a random variable:

$$
\int_{-\infty}^{\infty} P_{\xi^{*}} d x=\sum_{m=1}^{n} P\left(\xi^{*}=x_{m}\right)=z_{1}+\left(z_{2}-z_{1}\right)+\cdots+\left(z_{n}-z_{n-1}\right)+\left(1-z_{n}\right)=1
$$

Moreover $P_{\xi^{*}} \geq 0$ because

$$
\begin{array}{ll}
\forall_{x \in X} & P\left(\xi^{*}=x\right) \geq 0 \\
\forall_{x \notin X} & P\left(\xi^{*}=x\right)=0
\end{array}
$$

2. Let us notice, that the distribution function of $\xi^{*}$ has the following form:

$$
F_{\xi^{*}}\left(x_{m}\right)=z_{m}^{*}
$$

then, we have

$$
z_{m}^{*}=\min _{i \in I} z_{m}^{i}
$$

hence

$$
\forall_{m=1, \ldots, n} \quad z_{m}^{*} \leq z_{m}^{j}
$$

and

$$
\forall_{m=1, \ldots, n} \quad{ }^{(k)} z_{m}^{*} \leq{ }^{(k)} z_{m}^{j}
$$

which means that

$$
\xi_{i} \leq^{(k)} \xi^{*} .
$$

3. We will prove it, in the case of $k=1$ (analogously, it may be proved for larger $k$ )

From vectors $\mathbf{z}^{i}, \mathbf{z}^{j}$ definitions:

$$
\begin{aligned}
\mathbf{z}^{i} & =\left[F_{\xi_{i}}\left(x_{1}\right), F_{\xi_{i}}\left(x_{2}\right), \ldots, F_{\xi_{i}}\left(x_{n}\right)\right], \\
\mathbf{z}^{j} & =\left[F_{\xi_{j}}\left(x_{1}\right), F_{\xi_{j}}\left(x_{2}\right), \ldots, F_{\xi_{j}}\left(x_{n}\right)\right],
\end{aligned}
$$

and from $\xi_{i} \leq^{(1)} \xi_{j}$, we obtain

$$
\mathbf{z}^{i} \geq \mathbf{z}^{j}
$$

hence

$$
\mathbf{z}^{i}-\mathbf{z}^{*} \geq \mathbf{z}^{j}-\mathbf{z}^{*}
$$

Moreover we know from 2. that

$$
0 \leq \mathbf{z}^{i}-\mathbf{z}^{*} \text { and } 0 \leq \mathbf{z}^{j}-\mathbf{z}^{*}
$$


From the following properties of the augmented Tchebycheff norm

$$
\begin{aligned}
& 0 \leq \mathbf{u} \leq \mathbf{v} \quad \Rightarrow \quad\||\mathbf{u}|\|_{\infty}^{\lambda} \leq\||\mathbf{v}|\|_{\infty}^{\lambda}, \\
& \||\mathbf{u}|\|_{\infty}^{\lambda}=\||-\mathbf{u}|\|_{\infty}^{\lambda},
\end{aligned}
$$

finally, we obtain

$$
\left\|\left|\mathbf{z}^{*}-\mathbf{z}^{i}\right|\right\|_{\infty}^{\lambda} \geq\left\|\left|\mathbf{z}^{*}-\mathbf{z}^{j}\right|\right\|_{\infty}^{\lambda}
$$

4. It results immediately from monotonicity of the augmented Tchebycheff norm:

$$
0 \leq \mathbf{u} \leq \mathbf{v} \quad \Rightarrow \quad\||\mathbf{u}|\|_{\infty}^{\lambda} \leq\||\mathbf{v}|\|_{\infty}^{\lambda}
$$

5. Assuming positive values of $\rho>0$ and $\lambda>0$, we obtain strictly monotonicity of the augmented Tchebycheff norm:

$$
0 \leq \mathbf{u}<\mathbf{v} \Rightarrow\||\mathbf{u}|\|_{\infty}^{\lambda}<\||\mathbf{v}|\|_{\infty}^{\lambda}
$$

The above implication proves thesis 5 .

\section{References}

Ballestero, E. (1996). Selecting the CP metric: a risk aversion approach. European Journal of Operational Research, 97, 593-596.

Ballestero, E., \& Romero, C. (1993). Weighting in compromise programming: a theory on shadow process. Operations Research Letters, 13, 325-329.

Carrizosa, E., Conde, E., Pacual, A., \& Romero-Morales, D. (1996). Closest solutions in ideal-point methods. In R. Caballero, F. Ruiz, \& R. E. Steuer (Eds.), LNEMS: Vol. 455. Advances in multiple objective and goal programming (pp. 274-281). Berlin: Springer.

Copeland, T. E., \& Weston, J. F. (1988). Financial theory and corporate policy. Reading: Addison-Wesley.

Dentcheva, D., \& Ruszczyński, A. (2006). Inverse stochastic dominance constraints and rank dependent expected utility theory. Mathematical Programming Series B, 108, 297-311.

Gass, S. I., \& Roy, P. G. (2003). The compromise hypersphere for multiobjective linear programming. European Journal of Operational Research, 144, 459-479.

Gibbs, A. L., \& Su, F. E. (2002). On choosing and bounding probability metrics. International Statistical Review, 70(3), 419-435.

Kassem, M. (2008). Stability achievement scalarization function for multiobjective nonlinear programming problems. Applied Mathematical Modelling, 32(6), 1044-1055.

Kirkwood, C. W. (2002). Decision Tree Primer. Arizona State University. http://www.public.asu.edu/ $\sim$ kirkwood/DAStuff/decisiontrees.

Levy, H. (1992). Stochastic dominance and expected utility: survey and analysis. Management Science, 38, $553-593$.

Nowak, M. (2007). Aspiration level approach in stochastic MCDM problems. European Journal of Operational Research, 177, 1626-1640.

Ogryczak, W., \& Ruszczyński, A. (1997). From stochastic dominance to mean-risk models: semideviations as risk measures (Interim Report 97/027). International Institute for Applied Systems Analysis, Laxenburg, Austria.

Rolski, T. (1976). Order relations in the set of probability distributions and their applications in the queueing theory (Dissertation mathematicae 132). PAN, Warsaw.

Roman, D., Darby-Dowman, K., \& Mitra, G. (2006). Portfolio construction based on stochastic dominance and target return distributions. Mathematical Programming Series B, 108, 541-569.

Sitarz, S. (2008). Postoptimal analysis in multicriteria linear programming. European Journal of Operational Research, 191(1), 7-18.

Sitarz, S. (2010a). Dynamic programming with ordered structures: theory, examples and applications. Fuzzy Sets and Systems, 161, 2623-2641. 
Sitarz, S. (2010b). Standard sensitivity analysis and additive tolerance approach in MOLP. Annals of Operations Research, 181(1), 219-232.

Sitarz, S. (2012). Mean value and volume-based sensitivity analysis for Olympic rankings. European Journal of Operational Research, 216, 232-238.

Steuer, R. E., \& Choo, E. (1981). An interactive weighted Tchebycheff procedure for multiple objective programming. Mathematical Programming, 26, 326-344.

Stoyanov, S. V., Rachev, S. T., \& Fabozzix, F. J. (2012). Metrization of stochastic dominance rulet. International Journal of Theoretical and Applied Finance, 15(02), 1250017.

Trzaskalik, T., \& Sitarz, S. (2007). Discrete dynamic programming with outcomes in random variable structures. European Journal of Operational Research, 177(3), 1535-1548. 